ABSTRACT: Neuromuscular disorders frequently complicate sepsis and other critical illnesses in patients. Mice are the major species used as a model for sepsis. Nerve conduction studies (NCS), the primary tool for noninvasive assessment of nerve and muscle function, is challenging to perform in small animals. A reliable method for noninvasive, repeated NCS testing has not been reported in mice. We developed and validated a method for the repeated measurement of mouse sciatic nerve conduction in normal and septic mice. Our sedated and awake NCS system enabled minimally invasive long-term repeated measurements. The mean compound muscle action potential (CMAP) amplitude and latency were $17.4 \mathrm{mV}$ and $1.11 \mathrm{~ms}$, respectively $(n=59)$. There was an excellent intertester reproducibility by linear regression in both normal $(r=0.95)$ and septic $(r=0.98)$ mice. We also showed significant, time-dependent isoflurane-induced CMAP suppression in all animals, which was further exacerbated in septic mice. This study provides a new tool for the assessment of peripheral nerve/muscle function in mouse neuromuscular disease models that require repeated, long-term, and minimally invasive monitoring.

Muscle Nerve 40: 610-616, 2009

\title{
NONINVASIVE MODEL OF SCIATIC NERVE CONDUCTION IN HEALTHY AND SEPTIC MICE: RELIABILITY AND NORMATIVE DATA
}

\author{
MARCIN F. OSUCHOWSKI, DVM, PhD, ${ }^{1,3}$ JAMES TEENER, MD, ${ }^{2}$ and DANIEL REMICK, MD \\ ${ }^{1}$ Department of Pathology and Laboratory Medicine, Boston University School of Medicine, \\ Boston, Massachusetts, USA \\ ${ }^{2}$ Department of Neurology, University of Michigan Medical School, Ann Arbor, Michigan, USA \\ ${ }^{3}$ Ludwig Boltzmann Institute for Experimental and Clinical Traumatology, Vienna, Austria
}

Accepted 29 December 2008

Mice are the major species used as experimental models for various human diseases. This increasingly includes neuromuscular disorders such as neuromytonia, ${ }^{23}$ critical illness myopathy (CIM) ${ }^{18}$ amyotrophic lateral sclerosis, ${ }^{1}$ and Duchenne muscular dystrophy. ${ }^{19}$ Given the animal size, murine electrodiagnostic testing challenges investigators, and there is limited information regarding the normative (e.g., amplitude, latency) and intertester reliability and potential pitfalls of such experimentation. It is valuable to have a reliable measure of peripheral nerve and muscle function in the mouse. Such a stable and reproducible model is especially important in survival-type experiments, where

Abbreviations: CIM, critical-illness myopathy; CIP, critical-illness polyneuropathy; CLP, cecal ligation and puncture; CMAP, compound muscle action potential; NCS, nerve conduction studies

Key words: EMG; sciatic nerve conduction; normative data; reliability; longterm; isoflurane; sepsis; mouse

Correspondence to: D.G. Remick, currently at Department of Pathology and Laboratory Medicine, Boston University School of Medicine; 670 Albany Street, Biosquare III, Boston, MA 02118; e-mail: remickd@bu.edu

(C) 2009 Wiley Periodicals, Inc.

Published online 17 July 2009 in Wiley InterScience (www.interscience.wiley. com). DOI 10.1002/mus.21284 elucidation of the potential pathophysiology of a disease requires long-term assessment of nerve and muscle function at daily or weekly intervals without surgical preparation. This is pertinent to a number of neuromuscular diseases of unknown etiology including CIM and critical illness polyneuropathy (CIP). Both disorders frequently develop in critically ill ICU patients with the systemic inflammatory response syndrome (SIRS), usually associated with sepsis/septic shock. Whereas refined electrodiagnostic approaches such as stimulated single-fiber electromyography (EMG) are feasible in mice, ${ }^{6}$ they are generally not suitable for prolonged and repeated monitoring of nerve and muscle function.

A reliable, validated method for long-term, repeated assessment of peripheral nerve and muscle function has not been previously reported in mice. We sought to develop and standardize a method for repeated measurement of sciatic nerve conduction in outbred ICR mice and to determine the reference values and reliability of this system. We also assessed the effects of inhalation isoflurane anesthesia on the nerve/muscle-evoked potentials. To further verify 
the applicability of this technique in a severe disease model, we tested it in the cecal ligation and puncture (CLP) model of polymicrobial sepsis.

\section{MATERIALS AND METHODS}

Animals. Female ICR mice (Harlan Sprague-Dawley, Indianapolis, Indiana) with an average weight of $22 \mathrm{~g}$ (25-35 g; 5-7 weeks) were used in all experiments. The mice were acclimated to the laboratory environment for at least $48 \mathrm{~h}$ before measurements were taken and were kept in a temperature-controlled facility with a 12/12-h light/dark diurnal cycle after the measurements. All experiments were in accordance with the National Institutes of Health guidelines and the University of Michigan Animal Care and Use Committee.

Cecal Ligation and Puncture. To examine the reproducibility of sciatic nerve conduction studies in a severe disease model, recordings were also performed in CLP mice. This model is currently considered the gold standard for abdominal polymicrobial human sepsis. ${ }^{8,15}$ To generate $\approx 50 \%$ mortality during the early sepsis phase (first 5 days), an 18G needle and double cecal puncture were used. We followed the original CLP protocol introduced by Wichterman et al., ${ }^{20}$ with modifications. ${ }^{4}$ Specifically, all animals were resuscitated subcutaneously with warm saline (1 ml/animal) immediately postCLP, administered analgesic $(0.05 \mathrm{mg} / \mathrm{kg}$ b.w. buprenorphine; total of four subcutaneous (s.c.) injections every $12 \mathrm{~h}$ for 2 days) and given an antibiotic (25 mg/kg b.w. Imipenem; total of 10 s.c. injections every $12 \mathrm{~h}$ for 5 days) treatment. Buprenorphine was used exclusively for post-CLP analgesia and not for sedation purposes prior to nerve conduction studies (NCS). When tested in normal mice, buprenorphine did not influence the CMAP amplitude (data not shown).

Recordings. All animals were studied using a Nicolet (Madison, Wisconsin) Viking IV EMG machine. Prior to the initial measurements the electrode insertion site was shaved as depicted in Figure 1 and retrimmed as needed. Skin-surface temperature was maintained at $32-36^{\circ} \mathrm{C}$ by means of a heat lamp if necessary. Skin temperature was measured at the stimulation electrode insertion site by a Noncontact Infrared Thermometer (Fisher Scientific, Pittsburgh, Pennsylvania). Studies were performed both on sedated and awake animals to assess the potential impact of anesthesia on the recordings.

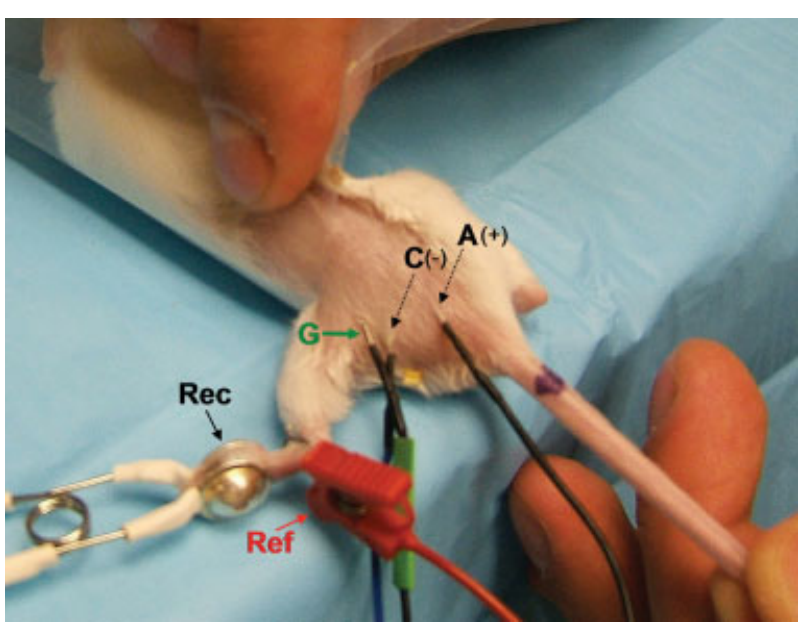

FIGURE 1. Minimally invasive NCS in the mouse. Prior to recording, lidocaine gel was applied over the stimulating needle insertion locations, the mouse was restrained in the decapicone, and the electrodes were attached as depicted on the picture. (A) stimulating needle anode; (C) stimulating cathode; $(\mathrm{G})$ needle ground electrode; (Ref) Meditrode clip reference electrode (Rec) silver cup earclip recording electrode. [Color figure can be viewed in the online issue, which is available at www.interscience.wiley.com]

Anesthetized Mice. Recordings in anesthetized mice were performed under isoflurane and typically lasted $\approx 3 \mathrm{~min}$ (excluding the induction phase). For induction, mice were sedated with $5 \%$ isoflurane in a plastic chamber and then placed outside for the NCS while the sedation with $3 \%$ isoflurane was continued via a rubber cone mask placed over the animals' heads. Recordings in a single sedated mouse lasted typically $2 \mathrm{~min}$ (including the period of electrode fitting) and never exceeded $3 \mathrm{~min}$.

Awake/Restrained. Animals who underwent NCS without anesthesia were placed in a plastic decapicone restrainer (Braintree Scientific, Braintree, Massachusetts), which was adapted to give free access to the hindlimb. Lidocaine gel was applied to the skin over the needle insertion locations $\approx 10 \mathrm{~min}$ prior to placing the stimulating electrodes. The mouse was restrained by one person while the second person positioned the electrodes and obtained recordings. Recordings in a single awake/restrained mouse typically lasted $3 \mathrm{~min}$ (including the period of electrode fitting) and never exceeded $5 \mathrm{~min}$.

Stimulation-Stimulus/Ground Electrodes. For stimulation, 1-cm stainless steel needle electrodes were placed subdermally for their full length: the anode just lateral to the midline, with the tip inserted $1 \mathrm{~mm}$ caudal to the tail-base and advanced parallel to the spine, and the cathode $\approx 3 \mathrm{~mm}$ lateral and parallel to 
the anode (Fig. 1). A 1-cm subdermal needle inserted in the proximal thigh served as the ground electrode. Each animal was stimulated with incremental impulse intensity until a maximal and artifact-free compound muscle action potential (CMAP) motor response was evoked. This was achieved typically over the range of currents from $30-80 \mathrm{~mA}$ and duration of $0.1-0.2 \mathrm{~ms}$.

Recording-Reference Electrodes. Recordings were obtained using a 9.5-mm silver cup earclip electrode (Electrode Store, Enumclaw, Washington). This electrode was minimally coated with an electrode gel and placed over the distal foot. This location likely resulted in recording from multiple small foot muscles. Two different reference electrodes were utilized: (1) a 1-cm stainless steel needle electrode that was inserted into the heel to a depth of $1-2 \mathrm{~mm}$, and (2) a small clip "Meditrode" delivery lead wire electrode (Life-Tech, Stafford, Texas). The Meditrode clip electrode had to be adapted for NCS use: the plastic body of the electrode was trimmed so that the roughly circular wire contacts were exposed. The contacts were bent slightly so that they made firm, but not crushing, contact when placed around the mouse ankle.

Statistics. For assessment of intertester reproducibility and the isoflurane effect in septic mice, recordings from the second day post-CLP were used. Normative data listed in the results are expressed as the mean \pm SD. The intertester and electrode correlation were analyzed by the linear regression and expressed as Pearson correlation coefficient $(r)$, linear equation slope, and 95\% confidence interval. Both the temporal changes in individual groups and intergroup differences were analyzed by analysis of variance (ANOVA) for repeated measures followed by either the Dunnett's Multiple Comparison Test (temporal intragroup changes) or Bonferroni posttest (temporal intergroup changes). $P<0.05$ was considered statistically significant. All analyses were performed using the Prism 3.0 program for Windows (GraphPad Prism, San Diego, California).

\section{RESULTS}

Setup. Figure 1 shows the awake/restrained recording setup with all attached electrodes. We only show the awake setup, since this requires specific restraining/positioning of animal body and extremities. The needle insertion area is shaved and the surface anesthetic gel (Lidocaine) is applied. The animal is then put into the plastic decapicone funnel up to

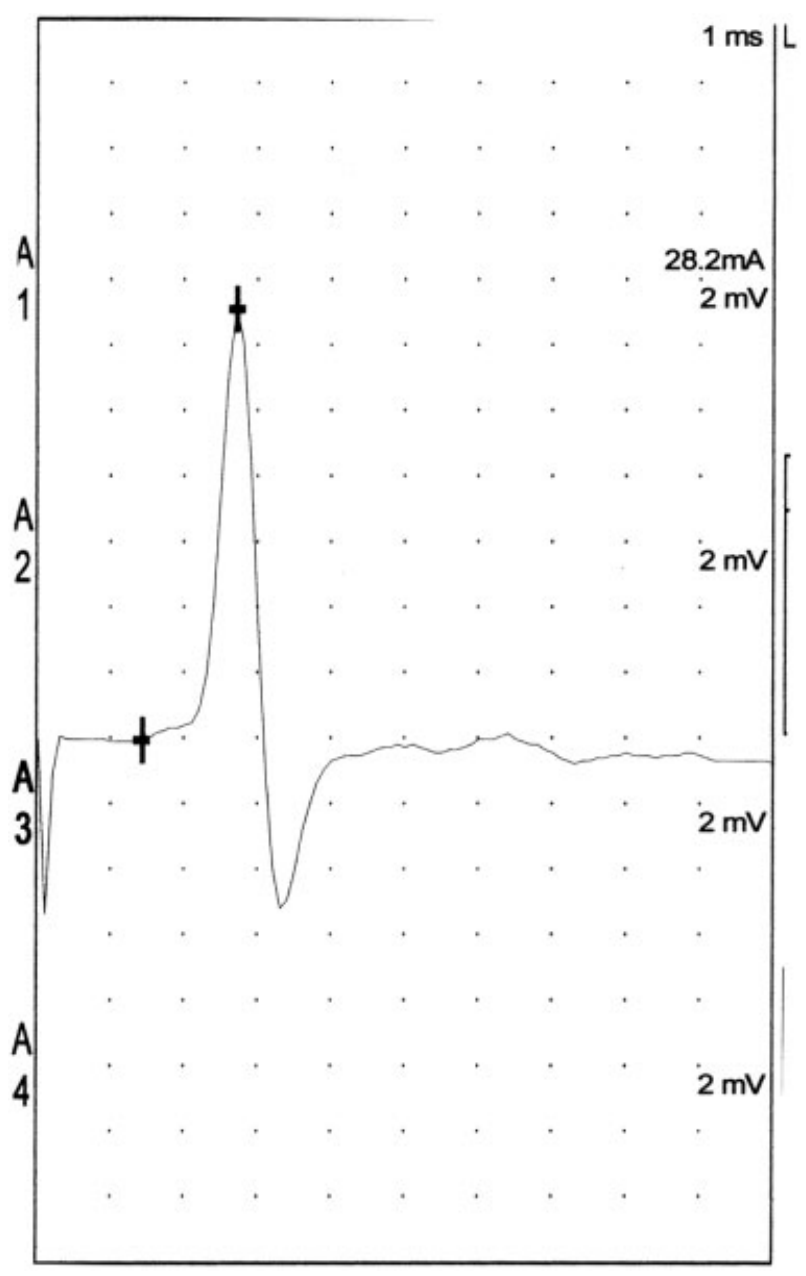

FIGURE 2. A representative biphasic waveform obtained using the described technique. Onset latency was measured from the initial negative deflection from baseline as marked. Minimal repositioning of the recording electrode was occasionally performed to minimize a positive deflection. Amplitude is measured from baseline to peak as marked by the plus signs.

$\approx 70 \%$ of its body length so that free access to the hindlimbs is provided. For the electrode fitting and recording the torso of the mouse needs to be immobilized and lightly stretched by gently pulling on the tail to prevent the mouse from hunching up. Figure 1 depicts the arrangement of the electrodes: stimulating (black dashed arrows) anode (A) and cathode $(\mathrm{C})$, ground (green arrow) electrode $(\mathrm{G})$, reference (red arrow) electrode (Ref), and recording (black solid arrow) electrode (Rec). The noninvasive and custom adapted "Meditrode" clip as a reference electrode is depicted in the picture.

Normative Data. A total of 59 normal mice underwent recording of sciatic nerve conduction. A representative CMAP waveform is depicted in Figure 2. 


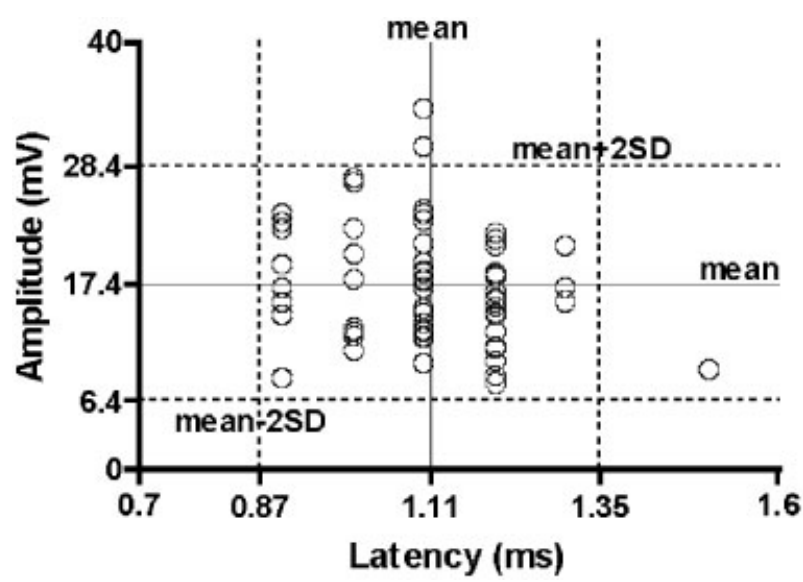

FIGURE 3. The normative distribution of CMAP amplitude ( $\mathrm{mV}, \mathrm{y}$ axis) and latency (ms, $x$ axis) values in the control mice $(n=59)$. Each circle represents an individual mouse.

The CMAP amplitude was measured from baseline to peak. The distal latency was measured as the initial deflection from baseline. Electrode placement was adjusted slightly to eliminate or minimize any initial positive deflection. The waveforms were biphasic with an initial negative deflection.

The mean of the recorded values for amplitude was $17.4 \pm 5.48 \mathrm{mV}$, and the latency was $1.11 \pm 0.12$ ms (Fig. 3). The lower limit of normal for the amplitude was $6.4 \mathrm{mV}$ and the upper limit of normal for latency was $1.35 \mathrm{~ms}$. For the $95 \%$ confidence interval (CI) of the mean, the lower limit was $16 \mathrm{mV}$ and 1.07 $\mathrm{ms}$, whereas the upper CI limit was $18.8 \mathrm{mV}$ and 1.14 ms (amplitude and latency, respectively). The coefficient of variation was $31 \%$ for amplitude and $11 \%$ for latency.

Intertester Reproducibility. To validate the intertester recording reproducibility, the NCS testing was performed independently by two different operators on the same set of either normal or septic mice $(n=$ 25 /group). After the first recording, all electrodes were removed and reapplied by the second operator prior to the second measurement. Figure 4 shows the linear regression plots listing the Pearson correlation coefficient $(r)$, regression line fitted through data points, and $95 \%$ CI. Recordings in normal mice revealed a strong intertester accuracy evidenced by a highly significant, positive correlation between the two datasets with $r=0.95$ and the linear equation slope $=0.94$ (Fig. 4A). The intertester recording reproducibility in septic mice was nearly ideal, with $r=0.98$ and slope $=0.96$ (Fig. 4B).

Temporal Reproducibility. Given that this application has been developed to allow repetitive measure- ments of nerve/muscle function, we tested the shortand long-term recording reproducibility in individual animals. The short-term monitoring did not cause any fluctuations in amplitude or latency. Figure $5 \mathrm{~A}$ depicts three examples of normal mice in which recordings were taken on 3 consecutive days. The latency values remained unaltered in virtually all
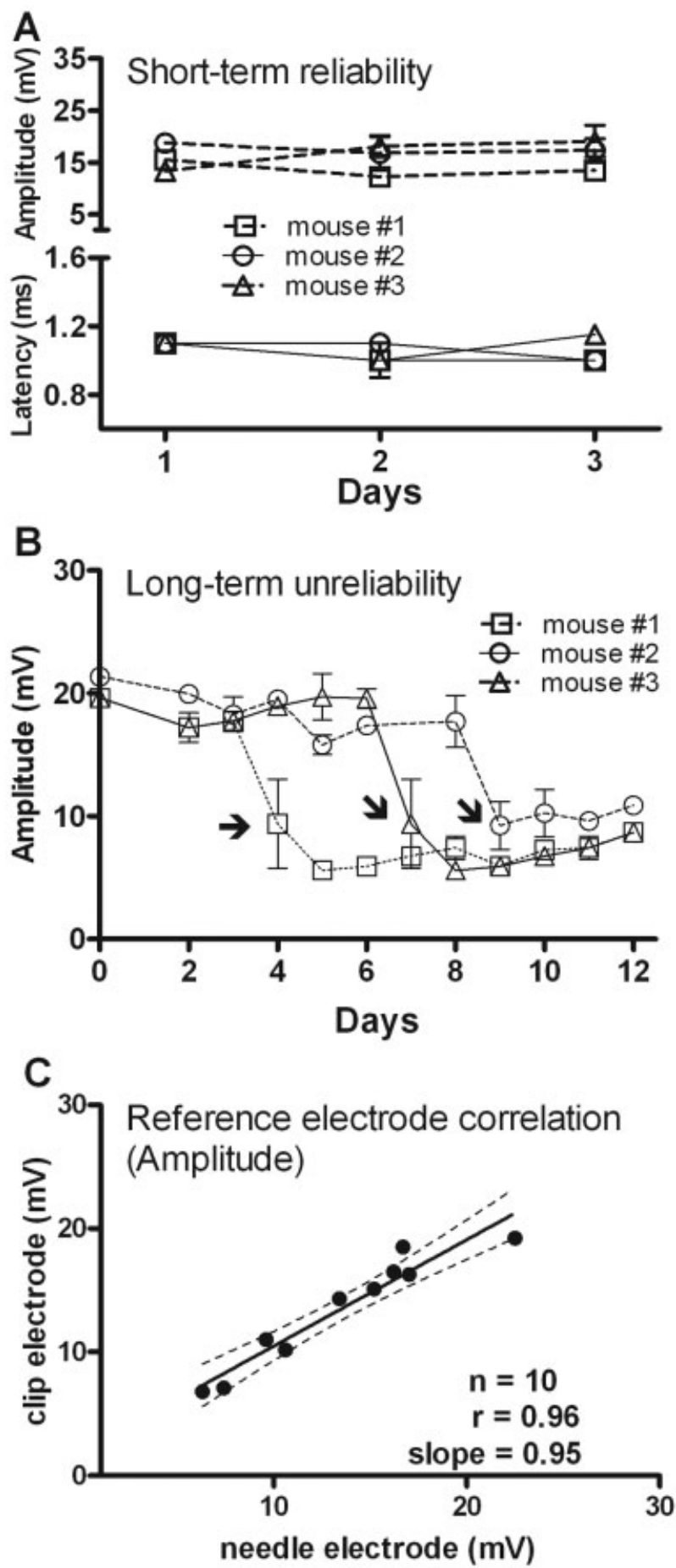
affected animals (not shown). However, repeated recordings with the heel needle electrode as a reference revealed occasional sharp decreases (typically exceeding $50 \%$ of the initial value) in the CMAP amplitude. This occurred randomly at later timepoints (typically after at least 3-4 initial repetitions) and was observed in $\approx 20 \%$ of tested animals (both normal and septic). Figure 5B depicts three normal mice in which an amplitude drop occurred between days 4-9. This effect was probably due to minor local soft tissue/nerve damage from repeated electrode insertions, as the recordings taken from the opposite limb were not reduced (data not shown). To eliminate the risk of potential injury during long-term repeated recordings, we adapted an alternative clip electrode (custom-made from "Meditrode" electrode; see Materials and Methods) to serve as a noninvasive reference, and compared its CMAP recording accuracy to the standard needle reference electrode. The clip and needle reference electrodes yielded virtually identical results, as evidenced by a nearly ideal positive correlation between the two datasets with $r=0.96$ and the linear equation slope $=0.95$ (Fig. 5C). This unexpected decrease in amplitude over time in some animals, likely due to injury related to electrode placement, highlights one of the challenges in performing electrodiagnostic studies in small animals.

Sedation-Dependent Depression. Finally, we investigated whether isoflurane anesthesia elicits a depressive effect on the CMAP in our sciatic nerve conduction model, either in normal or septic (CLP) mice. This is important since isoflurane is the inhalant of choice in most rodent survival surgery protocols and has been shown to modulate the CMAP in human

FIGURE 5. Temporal reliability in serial EMG recordings. (A) Short-term temporal profiles of CMAP amplitude and latency recorded over 3 consecutive days in three mice are plotted. (B) Long-term temporal profiles of CMAP amplitude and latency recorded for 12 consecutive days $(n=3)$ are plotted. Arrows indicate the first day on which a sudden amplitude drop in each individual mouse was recorded. This sudden CMAP amplitude drop was typically observed in $\approx 20 \%$ among all tested mice. (A,B) Data reported as mean \pm SEM of two repeated recordings taken by two different operators. (C) Linear regression plot for reference electrode correlation. Two consecutive recordings from the same mouse were taken by the same operator: the first with the clip (y axis) and the second with the needle (x axis) electrode. The EMG was performed in awake mice, and only the reference electrodes were changed during the test. All matched values were then analyzed by the linear regression for correlation. The graph shows the correlation coefficient, slope, regression line fitted through the data points $(n=10)$, and $95 \%$ confidence interval. patients. ${ }^{16,21}$ Immediately after induction of anesthesia, mice were placed on the table with a rubber cone mask (3\% isoflurane), and repeated $(0,1,2,3,5,8$, and $10 \mathrm{~min}$ ) recordings were made. Figure 6 depicts the temporal trajectory of CMAP amplitude change in both control and septic mice. The first recording taken immediately after induction of anesthesia (about 1 min duration) was designated as time zero. This was necessary, since the induction step is mandatory, and the time zero values in sedated animals were virtually identical to the values recorded prior to anesthesia using the awake/restrained setup (data not shown). In both groups $(n=5)$ there was a time-dependent reduction in CMAP amplitude. The decrease in amplitude was noted by the 3-min timepoint in both control (22\% decrease, $P<0.05)$ and septic (20\% decrease, $P>0.05)$ groups compared to
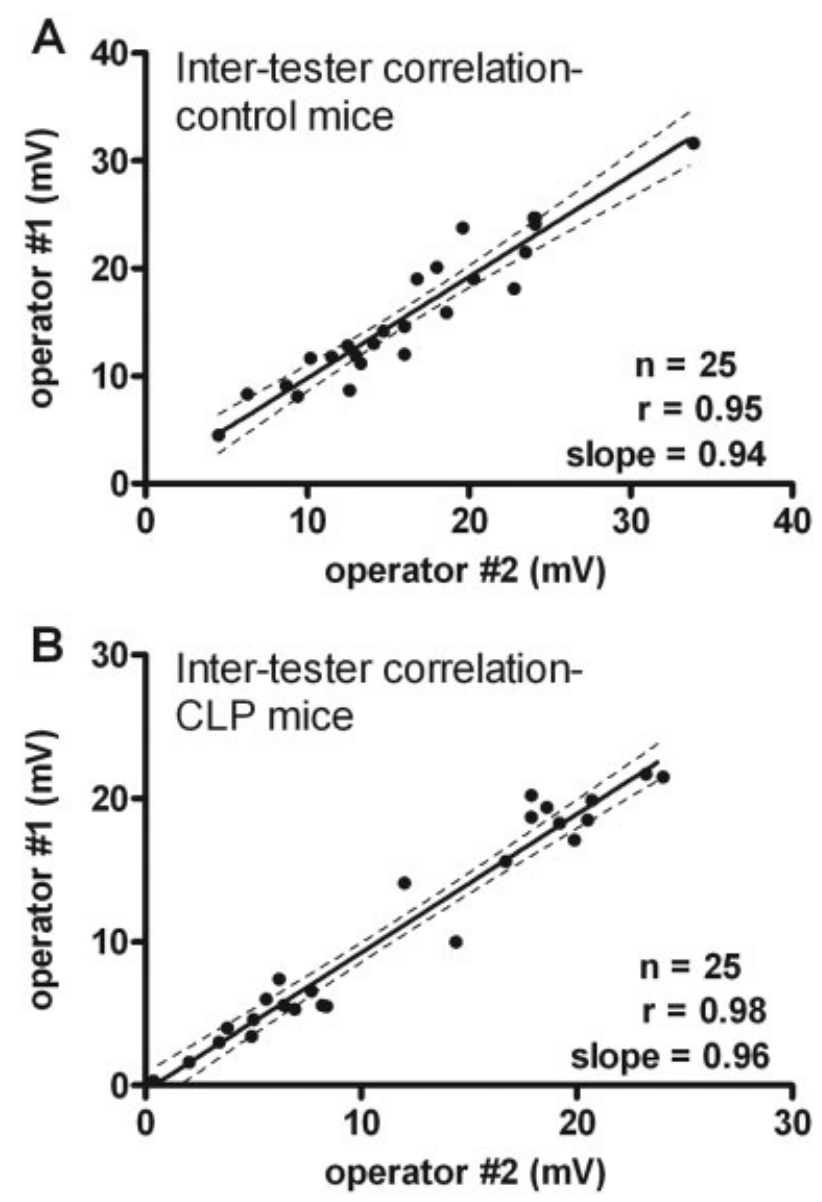

FIGURE 4. Linear regression plot for intertester correlation in control (A) and septic (B) mice. The first NCS recording was taken by operator no. 1 (y axis), then all electrodes were removed and reapplied by operator no. 2 for the second NCS measurement. All matched values were then analyzed by the linear regression for correlation. The graph shows correlation coefficient, slope, and regression line fitted through the data points $(n=$ 25 /group) as well as the $95 \%$ confidence interval. 


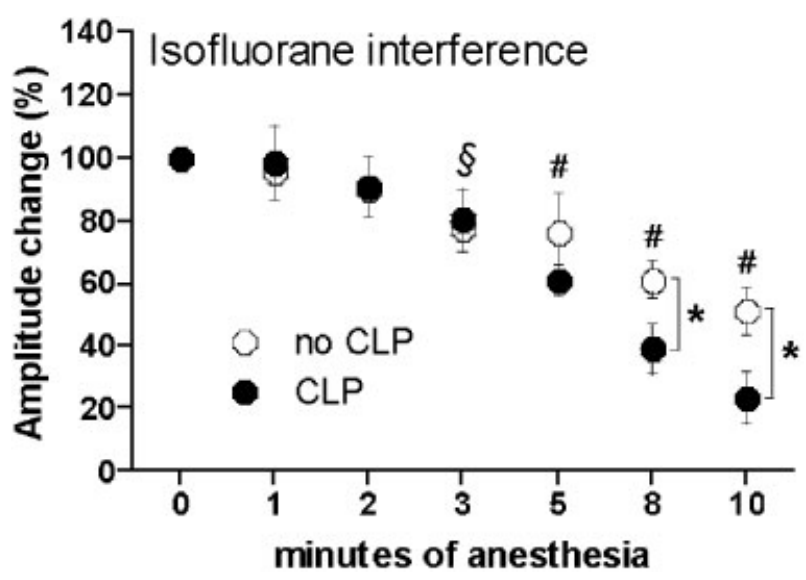

FIGURE 6. Temporal suppression of CMAP amplitude by isoflurane in control (no CLP) and septic (CLP) mice. Consecutive (0, $1,2,3,5,8$, and $10 \mathrm{~min}$ ) NCS were made in isoflurane-sedated control and septic mice $(n=5)$. Individual amplitude values $(\mathrm{mV})$ were normalized and expressed as percent change from the zero timepoint (y axis). Individual ( $n=5 /$ group) amplitude values (mA) were normalized and expressed as percent change from the zero timepoint. Data reported as mean \pm SEM. $\S P<0.05$ control versus time zero; $\# P<0.05$ control and septic versus time zero; ${ }^{*} P<0.05$ control versus septic.

the zero timepoint. While the isoflurane-induced reduction in CMAP amplitude progressed in subsequent timepoints $(P<0.05$ for CLP and no CLP), the rate of this decrease was more pronounced in the CLP mice, which was apparent by the 5 -min recording $(15 \%$ difference between control and CLP, $P<0.05)$. This effect increased further to result in a $28 \%$ difference at the 10-min timepoint $(P<0.05)$. These findings emphasize the importance of precise isoflurane dosing when the sedated protocol option is used for CMAP recordings. Excessive exposure to isoflurane can skew the study by depressing the CMAP and thereby producing false-positive results.

\section{DISCUSSION}

It is challenging to perform reproducible, reliable electrodiagnostic studies on small animals. Because one of our primary goals was to be able to perform repeated recordings over many days in different models of critical disease with significant early and late mortality, we developed a minimally invasive NCS setup. We chose to evaluate our NCS method in the CLP sepsis model, given that this model constitutes the best surrogate for abdominal polymicrobial human sepsis to date ${ }^{8,15}$ with sepsis deaths in both the acute and chronic phases. ${ }^{16,21}$ Additionally, critical-illness neuromuscular disorders (e.g., CIP and CIM) occur in up to $70 \%$ of septic patients. ${ }^{11,12}$ Other accurate electrodiagnostic methods, such as single-fiber $\mathrm{EMG}^{2,6}$ and multielectrode
EMG implants, ${ }^{3,9}$ have been described as useful in studying neuromuscular disorders related to sepsis. However, these methods are invasive or cumbersome for repeatedly evaluating neuromuscular function over many days. Our method, which utilized evoked muscle potentials triggered by the subdermal (needle) stimulation of sciatic nerve and recording from small foot muscles, displayed a high level of reliability and reproducibility while being sufficiently easy to perform to allow multiple recordings to be obtained over many days.

We tested a number of recording electrodes to optimize the quality of the recordings. Initial foot recordings from a $1-\mathrm{cm}$ subdermal needle or a 20-mm gelled silver/silver chloride skin adhesive electrode (Nicolet Biomedical) showed excessive interrecording variability. This was due to either variable amplitude depending on the insertion depth and focal injury to the paw (needle electrode) or poor adherence of the adhesive electrode after repeated use. The latter electrode was feasible if used for no more than three consecutive recordings. This was impractical due to the high costs of electrode replacement, and better reliability was achieved using the clip electrode. Reproducible recordings were obtained using a special-purpose 9.5-mm human silver cup spring earclip electrode. The electrode's discs were the appropriate size and could be custombent to provide optimal paw skin contact.

While the sedated preparation is far less demanding in terms of animal-handling skills, it requires extra caution with regard to the use of isoflurane. In our model, recordings made in the sedated state were not distorted and were virtually identical to the recordings taken in awake state, as long as the exposure to isoflurane did not exceed 3 min (Fig. 6). Inhaled isoflurane produces systemic analgesia and muscle relaxation. The mechanism by which it produces the anesthetic state is by disruption of synaptic transmission, since isoflurane binds to GABA, glutamate, and glycine receptors and impedes conduction in activated potassium channels. ${ }^{13}$ Although reported here for the first time in mice, the reduction of the CMAP caused by various volatile anesthetics has been observed in humans ${ }^{5,17}$ (isoflurane), cats $^{22}$ (various agents including isoflurane), and rats $^{7}$ (desflurane). This effect, regardless of the anesthetic used, is likely related to synaptic inhibition.

Although the sedated setup appears to be the method of choice if only a single operator is available, we discourage its use in other than control mice when recordings are collected at intervals shorter then $24 \mathrm{~h}$. In severely/acutely sick animals, such frequent sedation may have a detrimental ef- 
fect. Although we did not investigate the immediate recovery time after discontinuation of isoflurane, the sedation-dependant CMAP depression is likely transient, since the amplitude values taken $24 \mathrm{~h}$ after initial excessive sedation returned to normal in all control animals. Since in the CLP animals the combination of ketamine/xylazine induced significant hypothermia that frequently could not be corrected with the use of heat pads, ${ }^{10,14}$ we find its use inappropriate in such critical disease model(s) and did not test this type of anesthesia in our NCS setup. Other relevant inhalation anesthetics need to be tested to fully expose their potential modulatory effect on CMAP. Although this study is limited by only one substance tested, the reported depressive effect by isoflurane should alert investigators to this potential complication when isoflurane or alternative inhalants are to be used in this NCS model.

In case of a nonsedated recording, two operators are necessary: the first gently restrains the mouse while the second performs the recording. The applied grip needs to be adjusted carefully so mice remain both immobilized and calm during the recording. Excessive force provokes animals to fight back and may cause detachment of the recording electrode and difficulty in artifact-free recording. Our subjective observations showed that a short ( $\approx 60 \mathrm{~s})$ "acclimatization" (by unrestricted exploration) of mice to the environment prior to the recording made the procedure only marginally stressful to animals (almost no resistance). This was especially true in control animals, since awake recordings in mice post-CLP were typically uneventful and effortless given their poor physical condition.

This recording method allows repeated and longterm assessment of peripheral nerve and muscle function in individual mice without prior surgical preparation. It has proven to be useful when employed for the initial appraisal of motor function in different stages of experimental sepsis or other relevant critical diseases. When supplemented with other, more refined methods, the described NCS technique should prove an extremely valuable tool for the investigation of pathology in murine models of human diseases.

The work was partially supported by NIH grant GM 67189. Part of the data contained in the article was presented at the 53rd AANEM Scientific Meeting (Washington D.C., October, 2006) and received the President's Research Initiative Award.

\section{REFERENCES}

1. Benatar M. Lost in translation: treatment trials in the SOD1 mouse and in human ALS. Neurobiol Dis 2007;26:1-13.

2. Bolton CF, Young GB, Zochodne DW. The neurological complications of sepsis. Ann Neurol 1993;33:94-100.
3. Bolton CF. Neuromuscular manifestations of critical illness. Muscle Nerve 2005;32:140-163.

4. Ebong SJ, Call DR, Bolgos G, Newcomb DE, Granger JI, O'Reilly M, Remick DG. Immunopathologic responses to non-lethal sepsis. Shock 1999;12:118-126.

5. Golan DE, Tashjian AH Jr, Armstrong EJ, Armstrong AW (eds.). Principles of pharmacology: the pathophysiologic basis of drug therapy, 2nd ed. Baltimore: Lippincott Williams \& Wilkins; 2007. p 985.

6. Gooch CL, Mosier DR. Stimulated single fiber electromyography in the mouse: techniques and normative data. Muscle Nerve 2001;24:941-945.

7. Haghighi SS, Sirintrapun SJ, Keller BP, Oro JJ, Madsen R. Effect of desflurane anesthesia on transcortical motor evoked potentials. J Neurosurg Anesthesiol 1996;8:47-51.

8. Hubbard WJ, Choudhry M, Schwacha MG, Kerby JD, Rue LW III, Bland KI, Chaudry IH. Cecal ligation and puncture. Shock 2005;24(Suppl 1):52-57.

9. Meekins GD, Carter GT, Emery MJ, Weiss MD. Axonal degeneration in the Trembler-j mouse demonstrated by stimulated single-fiber electromyography. Muscle Nerve 2007;36:81-86.

10. Newsom DM, Bolgos GL, Colby L, Nemzek JA. Comparison of body surface temperature measurement and conventional methods for measuring temperature in the mouse. Contemp Top Lab Anim Sci 2004;43:13-18.

11. Osuchowski MF, Welch K, Siddiqui J, Remick DG. Circulating cytokine/inhibitor profiles reshape the understanding of the SIRS/CARS continuum in sepsis and predict mortality. J Immunol 2006;177:1967-1974.

12. Osuchowski MF, Welch K, Yang H, Siddiqui J, Remick DG. Chronic sepsis mortality characterized by an individualized inflammatory response. J Immunol 2007;179:623-630.

13. Pearson KG, Acharya H, Fouad K. A new electrode configuration for recording electromyographic activity in behaving mice. J Neurosci Methods 2005;148:36-42.

14. Remick DG, Xioa H. Hypothermia and sepsis. Front Biosci 2006;11:1006-1013.

15. Rittirsch D, Hoesel LM, Ward PA. The disconnect between animal models of sepsis and human sepsis. J Leukoc Biol 2007;81:137-143.

16. Rohde V, Krombach GA, Baumert JH, Kreit Schmann-Andermahr I, Weinzierl M, Gilsbach JM. Measurement of motor evoked potentials following repetitive magnetic motor cortex stimulation during isoflurane or propofol anaesthesia. $\mathrm{Br} \mathrm{J}$ Anaesth 2003;91:487-492.

17. Scholle HC, Biedermann F, Arnold D, Jinnah HA, Grassme R, Schumann NP. A surface EMG multi-electrode technique for characterizing muscle activation patterns in mice during treadmill locomotion. J Neurosci Methods 2005;146:174-182.

18. Teener JW, Rich MM. Dysregulation of sodium channel gating in critical illness myopathy. J Muscle Res Cell Motil 2006; 27:291-296.

19. Vainzof M, Yub-Guerrieri D, Onofre PC, Martins PC, Lopes VF, Zilberztajn D, Maia LS, Sell K, Yamamoto LU. Animal models for genetic neuromuscular diseases. J Mol Neurosci 2008;34:241-248.

20. Wichterman KA, Baue AE, Chaudry IH. Sepsis and septic shock-a review of laboratory models and a proposal. J Surg Res 1980;29:189-201.

21. Woodforth IJ, Hicks RG, Crawford MR, Stephen JP, Burke DJ. Variability of motor-evoked potentials recorded during nitrous oxide anesthesia from the tibialis anterior muscle after transcranial electrical stimulation. Anasth Analg 1996;82:744-749.

22. Yamada H, Transfeldt EE, Tamaki T, Nishiura H, Taylor BA, Torres F, Iaizzo PA. General anesthetic effects on compound muscle action potentials elicited by single or dual spinal cord stimulation. J Spinal Discord 1995;8:157-162.

23. Zielasek J, Toyka KV. Nerve conduction abnormalities and neuromyotonia in genetically engineered mouse models of human hereditary neuropathies. Ann N Y Acad Sci 1999;883: $310-320$. 